\title{
SINGULAR INTEGRAL EQUATIONS IN $L_{p}\left({ }^{1}\right)$
}

BY

\author{
HAROLD WIDOM
}

1. Introduction. In this paper we obtain some results concerning singular integral equations of the form

$$
K f(x) \equiv A(x) f(x)+\frac{B(x)}{\pi i} \int_{E} \frac{f(y)}{y-x} d y+M f(x)=g(x),
$$

where $E$ is a (measurable) subset of the reals of positive measure, $A(x)$ and $B(x)$ are (essentially) bounded on $E$, and $\boldsymbol{M}$ is a completely continuous operator on $L_{p}(E) ; g(x)$ is a given function in $L_{p}(E)$ and it is required to solve (1) for $f \in L_{p}(E)$.

The basic ideas involved are classical (see $[8$, part II $]$ ): the equivalence of the "dominant equation"

$$
K_{0} f(x) \equiv A(x) f(x)+\frac{B(x)}{\pi i} \int_{E} \frac{f(x)}{y-x} d y=g(x)
$$

with a Riemann-Hilbert problem

$$
G(x) f_{+}(x)=f_{-}(x)+h(x)
$$

(where $f_{+}(x)$ is the boundary function of a function analytic in $g_{z}>0$ and $f_{-}(x)$ the boundary function of a function analytic in $\left.g z<0\right)$; the solution of the Riemann-Hilbert problem by use of an appropriate factorization of $G$; the equivalence of (1) with a Fredholm equation. Pursuing these ideas for equations in $L_{p}$ the following question is seen to be important. For what weight functions $\omega(x)$ does an inequality hold of the form

$$
\int_{-\infty}^{\infty}|\tilde{f}(x)|{ }^{p} \omega(x) d x \leqq A \int_{-\infty}^{\infty}|f(x)|^{p} \omega(x) d x
$$

where $\tilde{f}(x)$ denotes the Hilbert transform of $f(x)$ ? Generally speaking it will turn out that the more weight functions one knows that satisfy (3), the greater is the class of singular integral equations which can be satisfactorily studied.

In $\$ 2$ we consider the above mentioned problem, together with a discussion of certain classes of functions, in preparation for the discussion of the Riemann-Hilbert problem in $\$ 3 . \S \S 4$ and 5 are devoted to the equation (2);

Presented to the Society, April 16, 1960; received by the editors September 8, 1959.

(1) This work was supported by a grant from the National Science Foundation. 
in particular for the operator $K_{0}$ in which $A \equiv 0$ and $B \equiv 1$ the spectrum may be found when $E$ is a finite union of (finite or infinite) intervals. In $\S 6$ we consider the reduction of (1) to a Fredholm equation.

2. Classes of functions. We introduce now certain classes of functions, defined on $(-\infty, \infty)$, which will be needed in the sequel. Throughout, we shall assume $1 \leqq p<\infty$, and $q=p /(p-1)$.

We denote by $L_{p}^{+}$the set of boundary functions of functions in $\mathfrak{W}_{p}$; thus $f \in L_{p}^{+}$means there is a function $\phi(z)$ analytic in $g z>0$ and satisfying

$1^{\circ} \int|\phi(x+i y)|^{p} d x \leqq A$ for $y>0$,

$2^{\circ} \lim _{y \rightarrow 0+} \phi(x+i y)=f(x)$ p.p.

We define $L_{p}^{-}$to be the set of complex conjugates of $L_{p}^{+}$functions. Clearly $f \in L_{p}^{-}$means there is a function $\psi(z)$ analytic in $g_{z}<0$ and satisfying

$3^{\circ} \int|\psi(x+i y)|^{p} d x \leqq A$ for $y<0$,

$4^{\circ} \lim _{y \rightarrow 0-} \psi(x+i y)=f(x)$ p.p.

The classes $L_{p}^{+}$and $L_{p}^{-}$have in common only the null function. For let $f \in L_{p}^{+} \cap L_{p}^{-}$with corresponding analytic functions $\phi, \psi$. Then for any finite interval $(a, b)$

$$
\lim _{y \rightarrow 0+} \int_{a}^{b}|\phi(x+i y)-\psi(x-i y)| d x=0
$$

(since $\phi(x+i y) \rightarrow f(x)$ in $L_{p}$ and $\psi(x-i y) \rightarrow f(x)$ in $L_{p}$ [3]), and so it follows from a theorem of Carleman [1, Theorem II, p. 40] that $\phi$ and $\psi$ are analytic continuations of each other and together represent an entire function $f(z)$. From the Cauchy integral formula we derive the inequality

$$
|f(z)| \leqq \frac{1}{\pi R^{2}} \iint|f(x+i y)| d x d y
$$

where the region of integration is a disc with center $z$ and radius $R$. It follows from (4), using $1^{\circ}$ and $3^{\circ}$ above, that $f(z)$ is bounded, and so is a constant. Since $f \in L_{p}$ this constant must be zero.

Given a function $f$ such that $f(x) /(1+|x|) \in L_{1}$ the principal value integral

$$
\frac{1}{\pi} \int \frac{f(y)}{y-x} d y
$$

exists p.p. and so we may define the two functions

$$
\begin{aligned}
& f_{+}(x)=\frac{1}{2 \pi i} \int \frac{f(y)}{y-x} d y+\frac{1}{2} f(x), \\
& f_{-}(x)=-\frac{1}{2 \pi i} \int \frac{f(y)}{y-x} d y+\frac{1}{2} f(x) .
\end{aligned}
$$

We use the subscripts + and - advisedly since if $f \in L_{p}$ and $p>1$ then 
$f_{+} \in L_{p}^{+}\left[3\right.$, Theorem 3.1] and $f_{-} \in L_{p}^{-} ;$moreover the operators $f \rightarrow f_{+}$and $f \rightarrow f_{-}$are bounded when $p>1$, as is immediate from the theorem of M. Riesz. If $f \in L_{p}^{+}$then $f_{+}=f$ and $f_{-}=0$; if $f \in L_{p}^{-}$then $f_{-}=f$ and $f_{+}=0$. Assuming $f \in L_{p}^{+}$ with corresponding analytic function $\phi(z)$ we have

$$
\phi(x+i y)=\frac{1}{2 \pi i} \int \frac{f(u)}{u-x-i y} d u \rightarrow f_{+}(x)
$$

as $y \rightarrow 0+$. On the other hand $\phi(x+i y) \rightarrow f(x)$, and so $f=f_{+}$. Therefore also $f_{-}=f-f_{+}=0$. The corresponding results for $L_{p}^{-}$follow by taking complex conjugates.

The classes $L_{p}^{+}$and $L_{p}^{-}$are closed in $L_{p}$. We prove this only for $p>1$, which is the case we shall need. Let $f_{n} \in L_{p}^{+}, f_{n} \rightarrow f$ in $L_{p}$ mean. Then $f_{n}=\left(f_{n}\right)_{+} \rightarrow f_{+}$ in mean. Therefore $f=f_{+}$and so $f \in L_{p}^{+}$.

We next define certain classes of functions larger than $L_{p}, L_{p}^{+}$, and $L_{p}^{-}$:

$$
\begin{aligned}
& f \in \mathfrak{R}_{p} \Leftrightarrow \frac{f(x)}{1+|x|} \in L_{p}, \\
& f \in \mathfrak{l}_{p}^{+} \Leftrightarrow \frac{f(x)}{x+i} \in L_{p}^{+}, \\
& f \in \mathfrak{R}_{p}^{-} \Leftrightarrow \frac{f(x)}{x-i} \in L_{p}^{-} .
\end{aligned}
$$

It is easily seen that for $p>1$, if $f \in L_{p}$ (resp. $L_{p}^{+}, L_{p}^{-}$) and $g \in \mathbb{R}_{q}$ (resp. $\mathbb{R}_{q}^{+}, \mathbb{R}_{q}^{-}$) then $f g \in \mathfrak{R}_{1}$ (resp. $\mathfrak{R}_{1}^{+}, \mathfrak{R}_{1}^{-}$).

Given a function $f$ such that $f(x) /\left(1+x^{2}\right) \in L_{1}$ the principal value integral

$$
\int \frac{1+y x}{y-x} \frac{f(x)}{1+y^{2}} d y
$$

exists p.p., and so we may define the two functions

$$
\begin{aligned}
& f_{\pi}(x)=\frac{1}{2 \pi i} \int \frac{1+y x}{y-x} \frac{f(y)}{1+y^{2}} d y+\frac{1}{2} f(x), \\
& f_{\mu}(x)=-\frac{1}{2 \pi i} \int \frac{1+y x}{y-x} \frac{f(y)}{1+y^{2}} d y+\frac{1}{2} f(x)
\end{aligned}
$$

In particular $f_{\pi}$ and $f_{\mu}$ are defined if $f \in \mathbb{R}_{p}$. If $f \in L_{p}$ then $f_{\pi}=f_{+}+$const and $f_{\mu}=f_{-}+$const. This is clear since

$$
\frac{1+y x}{y-x} \frac{1}{1+y^{2}}=\frac{1}{y-x}-\frac{y}{1+y^{2}} .
$$

If $f \in R_{p}^{+}$then $f_{\pi}=f$ and $f_{\mu}=0$; if $f \in R_{p}^{-}$then $f_{\mu}=f$ and $f_{\pi}=0$. Assume first that 
$f(x)$ is the restriction to the real line of a function $f(z)$ such that $g(z)=f(z) /(z+i)$ is analytic in $g z>-\delta(\delta>0)$ and satisfies there a bound

$$
\int|g(x+i y)|^{p} d x \leqq A
$$

Then we have, for $g z>0$,

$$
f(z)=\frac{1}{2 \pi i} \int_{-\infty}^{\infty} \frac{1+u z}{u-z} \frac{f(u)}{1+u^{2}} d u .
$$

Consequently, setting $z=x+i y$ and letting $y \rightarrow 0+$, we have

$$
f(z)=\frac{1}{2 \pi i} P V \int_{-\infty}^{\infty} \frac{1+u x}{u-x} \frac{f(u)}{1+u^{2}} d u+\frac{1}{2} f(x)=f_{\pi}(x) .
$$

For general $f \in R_{p}^{+}$, set $f_{n}(x)=\phi(x+i / n)$, where $\phi$ is the function analytic in $g_{z}>0$ of which $f$ is the boundary function. Then $f_{n}=\left(f_{n}\right)_{\pi}$, and so

$$
f-f_{\pi}=\lim _{n \rightarrow \infty}\left(f_{n}-f\right)_{\pi}
$$

almost everywhere, where $n$ runs through an appropriate sequence of integers. Let $(-a, a)$ be a fixed interval and assume $x \in(-a, a)$ is such that $f(x)$ $=\lim f_{n}(x)$. We have

$$
\begin{aligned}
\left(f_{n}-f\right)_{\pi}(x)= & \frac{1}{2 \pi i} \int_{-2 a}^{2 a} \frac{1+y x}{y-x} \frac{f_{n}(y)-f(y)}{1+y^{2}} d y \\
& +\frac{1}{2 \pi i} \int_{|y|>2 a} \frac{1+y x}{y-x} \frac{f_{n}(y)-f(y)}{1+y^{2}} d y+\frac{1}{2}\left\{f_{n}(x)-f(x)\right\} \\
= & I_{1}+I_{2}+\frac{1}{2}\left\{f_{n}(x)-f(x)\right\} .
\end{aligned}
$$

For $n$ sufficiently large the last term in (6) can be made less than $\epsilon$, and so can $I_{2}$ since $|x| \leqq a$. On the other hand

$$
I_{1}=\frac{1}{2 \pi i} \int_{-2 a}^{2 a} \frac{f_{n}(y)-f(y)}{y-x} d y-\frac{1}{2 \pi i} \int_{-2 a}^{2 a} \frac{y}{1+y^{2}}\left\{f_{n}(y)-f(y)\right\} d y
$$

and the second term on the right of (7) tends to zero as $n \rightarrow \infty$. Also, since $f_{n} \rightarrow f$ in $L_{1}$ on $(-2 a, 2 a)$, the first term on the right of (7) tends to zero in $L_{r}(-2 a, 2 a)$ for any $r<1$ (this follows from the proof of Theorem 105 of [11]), so by choosing an appropriate sequence of $n$ 's will tend to zero almost everywhere in $(-2 a, 2 a)$. We have therefore shown that $f-f_{\pi}=\lim \left(f_{n}-f\right)_{\pi}$ is zero p.p. in any finite interval, and so it is zero almost everywhere. Thus $f=f_{\pi}$; and we also have $f_{\mu}=f-f_{\pi}=0$. Similarly $f \in \mathfrak{R}_{p}^{-}$implies $f_{\mu}=f$ and $f_{\pi}=0$. 
Added in proof. An additive constant must be inserted in the right side of $\left(5^{\prime}\right)$. Thus various statements concerning $\pi$ and $\mu$ are incorrect and must be modified. The reader will see that this causes little difficulty.

Finally we note that the only functions common to $\mathfrak{R}_{p}^{+}$and $\mathfrak{R}_{p}^{-}$are the constants, and if $p=1$ the constant must be zero. The proof of this is similar to that of the analogous result concerning $L_{p}^{+}$and $L_{p}^{-}$given above, and is therefore omitted.

We come now to the problem concerning weight functions for Hilbert transforms mentioned in the introduction. The Hilbert transform of $f(x)$ is the function $\tilde{f}(x)$ defined by (5). Let $E$ and $F$ be two sets of positive measure, $p>1$. Let $\rho(x)$ be a function defined on $(-\infty, \infty)$ which is finite and nonzero almost everywhere. We say that $\rho$ belongs to the class $W_{p, E, F}$ if an inequality holds of the form

$$
\int_{F}|\rho(x) \tilde{f}(x)|^{p} d x \leqq A^{p} \int_{E}|\rho(x) f(x)|^{p} d x
$$

(where $A$ is independent of $f$ ) for all $f$ satisfying

(a) $f=0$ outside $E$,

(b) $f(x) /(1+|x|) \in L_{1}$.

By a simple approximation argument, which we omit, it is easily seen that the class of $f^{\prime}$ 's for which (8) is required to hold can be narrowed considerably. In fact, (b) can be replaced by

$\left(b^{\prime}\right) f$ vanishes outside a finite interval, and both $f$ and of are bounded.

Lemma 1. If $\rho \in W_{p, E, F}$ then $\rho^{-1} \in W_{q, F, E}$.

Proof. Assume $g$ vanishes outside $F$ and outside a finite interval, and that $g$ and $\rho^{-1} g$ are bounded. We want to prove an inequality

$$
\int_{E}\left|\rho^{-1}(x) \tilde{g}(x)\right|^{q} d x \leqq A^{q} \int_{F}\left|\rho^{-1}(x) g(x)\right|^{q} d x .
$$

It suffices to prove

$$
\left|\left(\rho^{-1} \tilde{g}, f\right)\right| \leqq A\left\|^{-1} g\right\|_{q}\|f\|_{p}
$$

for all $f$ vanishing outside $E$ and outside a finite interval, and such that $f$ and $\rho^{-1} f$ are bounded. But for such $f$,

$$
\begin{aligned}
\left|\left(\rho^{-1} \tilde{g}, f\right)\right| & =\left|\left(\tilde{g}, \bar{\rho}^{-1} f\right)\right|=\left|\left(g,\left(\bar{\rho}^{-1} f\right) \sim\right)\right| \\
& \left.=\mid\left(\rho^{-1} g, \bar{\rho}^{(} \overline{\boldsymbol{\rho}}^{-1} f\right) \sim\right) \mid \leqq A\left\|\rho^{-1} g\right\|_{q}\|f\|_{p},
\end{aligned}
$$

the last inequality holding since $\bar{\rho} \in W_{p, E, F}$.

Lемма 2. If $\rho \in W_{p, E, F}$ then 


$$
\int_{F} \frac{|\rho(x)|^{p}}{1+|x|^{p}} d x<\infty, \quad \int_{E} \frac{|\rho(x)|^{-q}}{1+|x|^{q}} d x<\infty .
$$

Proof. Let $E^{\prime}$ be a subset of $E$ of positive measure on which $\rho$ is bounded. Find points $\alpha, \beta$ and a positive $\epsilon$ so that the sets

$$
E_{1}=E^{\prime} \cap(\alpha-\epsilon, \alpha+\epsilon), \quad E_{2}=E^{\prime} \cap(\beta-\epsilon, \beta+\epsilon)
$$

both have positive measure, and so that in addition the intervals $(\alpha-2 \epsilon, \alpha+2 \epsilon)$ and $(\beta-2 \epsilon, \beta+2 \epsilon)$ are disjoint. Let $f_{i}$ be the characteristic function of $E_{i}(i=1,2)$. Then for some $a>0$ we have $\left|\tilde{f}_{1}(x)\right| \geqq a|x-\alpha|^{-1}$ for $|x-\alpha| \geqq 2 \epsilon$, so

$$
\int_{x \in F ;|x-\alpha| \geqq 2 \epsilon} \frac{|\rho(x)|^{p}}{|x-\alpha|^{p}} d x<\infty .
$$

Similarly $\left|\tilde{f}_{2}(x)\right| \geqq a|x-\beta|^{-1}$ for $|x-\beta| \geqq 2 \epsilon$, so

$$
\int_{x \in F ;|x-\beta| \geq 2 \epsilon} \frac{|\rho(x)|^{p}}{|x-\beta|^{p}} d x<\infty .
$$

From (9) and (10) and the fact that $(\alpha-2 \epsilon, \alpha+2 \epsilon)$ and $(\beta-2 \epsilon, \beta+2 \epsilon)$ are disjoint follows the first conclusion of the lemma. The second follows from the first, by Lemma 1.

Lemma 3. If $\operatorname{dist}(E, F)>0$ and at least one of the sets is bounded, the conditions of Lemma 2 are sufficient to insure that $\rho$ belong to $W_{p, E, F}$.

Proof. If $x \in F, y \in E$ we have

$$
\frac{1}{|y-x|} \leqq \frac{A}{(1+|x|)(1+|y|)}
$$

for a suitable constant $A$. Therefore if $f=0$ outside $E$,

$$
\begin{aligned}
\int_{F}|\rho(x) \tilde{f}(x)|^{p} & \leqq A \int_{F} \frac{|\rho(x)|^{p}}{1+|x|^{p}} d x\left\{\int_{E} \frac{|f(y)|}{1+|y|} d y\right\}^{p} \\
& \leqq A\left\{\int_{E} \frac{\left|\rho^{-1}(y)\right||\rho(y) f(y)|}{1+|y|} d y\right\}^{p} \\
& \leqq A\left\{\int_{E} \frac{\left|\rho^{-1}(y)\right|^{q}}{1+|y|^{q}} d y\right\}^{p / q} \int_{E}|\rho(y) f(y)|^{p} d y \\
& \leqq A \int_{E}|\rho(y) f(y)|^{p} d y
\end{aligned}
$$

(where of course we have used " $A$ " to represent several different constants). This proves the lemma. 
The next lemma, which is an extension of the classical M. Riesz theorem that $\|\tilde{f}\|_{p} \leqq A\|f\|_{p}$, is also a partial converse of Lemma 2 . We write $W_{p}$ for $W_{p, E, F}$ when $E=F=(-\infty, \infty)$.

Lemma 4. Let $\rho \in R_{p}^{+}$and assume that $\operatorname{sgn} \rho^{p}$ takes on at most two values, not negatives of each other. Assume in addition that $p$ is an even integer. Then $\rho$ belongs to $W_{p}$.

Proof. It suffices to prove an inequality $\|\rho \tilde{f}\|_{p} \leqq A\left\|_{\rho f}\right\|_{p}$ for $f$ real, continuously differentiable, and vanishing outside a finite interval; a simple argument will then yield the inequality for all $f$ satisfying condition (b) above. With these restrictions on $f$ define, for $z=x+i y$ and $y>0$,

$$
\Phi(z)=\frac{1}{\pi i} \int \frac{f(t)}{t-z} d t=U(x, y)+i V(x, y),
$$

$U$ and $V$ being real. We have then an inequality

$$
|\Phi(z)| \leqq \frac{A}{1+|z|}
$$

and moreover

$$
\lim _{y \rightarrow 0+} U(x, y)=f(x), \quad \lim _{y \rightarrow 0+} V(x, y)=-\tilde{f}(x) .
$$

For any $\epsilon>0$ we have

$$
\int_{-\infty}^{\infty} \rho(x)^{p} \Phi(x)^{p} \frac{d x}{(x+i)^{\epsilon}}=\lim _{y \rightarrow 0+} \int_{-\infty+i y}^{\infty+i y} \rho(z)^{p} \Phi(z)^{p} \frac{d z}{(z+i)^{\epsilon}}
$$

(where, for $g z \geqq 0$, we take $0<\arg (z+i)<\pi)$. This is easily verified if we use the bound (11) and the fact that

$$
\lim _{y \rightarrow 0+} \int_{-\infty}^{\infty} \frac{\left|\rho(x+i y)^{p}-\rho(x)^{p}\right|}{1+x^{p}} d x=0 .
$$

Now the integral on the right of (12) is clearly independent of $y$, and since

$$
\int_{-\infty+i y}^{\infty+i y}|\rho(z) \Phi(z)|^{p} d z
$$

is bounded in $y$, the integral on the right of $(12)$ is $O\left(y^{-c}\right)$ as $y \rightarrow \infty$. It follows that

$$
\int_{-\infty}^{\infty} \rho(x)^{p} \Phi(x)^{p} \frac{d x}{(x+i)^{e}}=0
$$

Now letting $\epsilon \rightarrow 0$ under the integral sign, as we may by the theorem on dominated convergence, we deduce 


$$
\int_{-\infty}^{\infty} \rho(x)^{p} \Phi(x)^{p} d x=0
$$

Consequently

$$
\begin{aligned}
0 & =\int \rho(x)^{p}\{\tilde{f}(x)+i f(x)\}^{p} d x \\
& =\int \rho(x)^{p} \tilde{f}(x)^{p} d x+\sum_{n=1}^{p}\left(\begin{array}{l}
p \\
n
\end{array}\right) i^{n} \int \rho(x)^{p} \tilde{f}(x)^{p-n} f(x)^{n} d x .
\end{aligned}
$$

Applying Hölder's inequality to each integral under the summation sign, we obtain the inequality

$$
\begin{aligned}
& \left|\int \rho(x)^{p} \tilde{f}(x)^{p} d x\right| \\
& \qquad A \sum_{n=1}^{p}\left\{\int|\rho(x) f(x)|^{p} d x\right\}^{n / p}\left\{\int|\rho(x) \tilde{f}(x)|^{p} d x\right\}^{(p-n) / p}
\end{aligned}
$$

Now by our assumption concerning sgn $\rho^{p}$, and the fact that $\tilde{f}(x)^{p} \geqq 0$, it is clear that we can find $\nu_{1}, \nu_{2}$ (independently of $f$ ) so that

$$
\nu_{1} \leqq \arg \{\rho(x) f(x)\}^{p} \leqq \nu_{2}, \quad \nu_{2}-\nu_{1}<\pi .
$$

We have then

$$
|\rho(x) \tilde{f}(x)|^{p} \leqq \sec \frac{1}{2}\left(\nu_{2}-\nu_{1}\right) R e^{(-i / 2)\left(\nu_{1}+\nu_{2}\right)}\{\rho(x) \tilde{f}(x)\}^{p}
$$

and so

$$
\int|\rho(x) \tilde{f}(x)|^{p} d x \leqq A\left|\int \rho(x)^{p} \tilde{f}(x)^{p} d x\right|
$$

Thus (13) gives

$$
X^{p} \leqq A \sum_{n=1}^{p} X^{p-n}
$$

where we have set $X=\|\rho \tilde{f}\|_{p} /\|\rho f\|_{p}$. It follows that $X$ is bounded.

We can now produce many functions belonging to $W_{p}$. We shall need an interpolation theorem due to E. M. Stein [9, Theorem 2]:

Let $T$ be a linear transformation from simple functions on one measure space to measurable functions on another. Suppose $1 \leqq r_{1}, r_{2}, s_{1}, s_{2} \leqq \infty$ and $r^{-1}$ $=(1-t) r_{1}^{-1}+t r_{2}^{-1}, s^{-1}=(1-t) s_{1}^{-1}+t s_{2}^{-1}$, where $0 \leqq t \leqq 1$. Suppose that for simplef

and

$$
\left\|(T f) \cdot k_{1}\right\|_{\rho_{1}} \leqq M_{1}\left\|f \cdot u_{1}\right\|_{r}
$$




$$
\left\|(T f) \cdot k_{2}\right\|_{\Omega_{2}} \leqq M_{2}\left\|f \cdot u_{2}\right\|_{r_{2}} .
$$

Let $k=k_{1}^{1-t} k_{2}^{2}, u=u_{1}^{1-t} u_{2}^{2}$. Then we have

$$
\|(T f) \cdot k\|_{\bullet} \leqq M_{t}\|f \cdot u\|_{r}
$$

with $M_{t}=M_{1}^{1-t} M_{2}^{t}$.

In our applications of Stein's theorem $T f$ will be $\tilde{f}$ and $r=s, r_{1}=s_{1}, r_{2}=s_{2}$.

LEMмA 5. Let $E$ be a set of reals and define

$$
\rho(x)=\exp \left\{\alpha \int_{E} \frac{1+y x}{y-x} \frac{d y}{1+y^{2}}+\pi i \alpha \chi_{E}(x)\right\} .
$$

Then if $|\alpha|<\min \left(p^{-1}, q^{-1}\right)$ we have $\rho \in R_{p}^{+} \cap W_{p}$. (Here $\chi_{E}$ denotes the characteristic function of $E$.)

Proof. Let $f$ be a real bounded function, and consider the analytic function

$$
\Phi(z)=\frac{1}{\pi} \int_{-\infty}^{\infty} \frac{1+u z}{u-z} \frac{f(u)}{1+u^{2}} d u, \quad g z>0 .
$$

Making the changes of variable

$$
\begin{array}{ll}
u=\tan \frac{\theta}{2}, & f(u)=g(\theta), \\
z=i \frac{1-\zeta}{1+\zeta}, & \Phi(z)=\Psi(\zeta),
\end{array}
$$

we obtain after a simple computation

$$
\Psi(\zeta)=\frac{i}{2 \pi} \int_{-\pi}^{\pi} \frac{e^{i \theta}+\zeta}{e^{i \theta}-\zeta} g(\theta) d \theta, \quad|\zeta|<1 .
$$

Let $g(\theta)$ have the Fourier series $(1 / 2) a_{0}+\sum\left(a_{n} \cos n \theta+b_{n} \sin n \theta\right)$. Then defining

$$
\begin{aligned}
& g(r, \theta)=\frac{1}{2} a_{0}+\sum_{n=1}^{\infty} r^{n}\left(a_{n} \cos n \theta+b_{n} \sin n \theta\right), \\
& \bar{g}(r, \theta)=\sum_{n=1}^{\infty} r^{n}\left(a_{n} \sin n \theta-b_{n} \cos n \theta\right),
\end{aligned}
$$

we see that

$$
\Psi\left(r e^{i \theta}\right)=i g(r, \theta)-\bar{g}(r, \theta), \quad r<1 .
$$

Now there is a theorem of Zygmund [12] (or [13, ex. 3 of $\S 7.6]$ ) to the effect that if $|g(\theta)| \leqq 1$ then 


$$
\frac{1}{2 \pi} \int_{-\pi}^{\pi} e^{ \pm(\pi / 2-\epsilon) \vec{\sigma}(r, \theta)} d \theta \leqq \csc \epsilon
$$

for any $\epsilon>0$. It follows that if $|g(\theta)| \leqq \pi /(2 p+\delta)(\delta>0)$ then

$$
\int_{-\pi}^{\pi}\left|e^{p \Psi\left(r e^{i \theta}\right)}\right| d \theta \leqq A, \quad r<1 .
$$

If we now change variables back by (15) we obtain

$$
\int_{-\infty}^{\infty} \frac{\left|e^{p \Phi(x+i y)}\right|}{1+x^{2}} d x \leqq A, \quad y>0,
$$

if $|f(x)| \leqq \pi /(2 p+\delta)$. In particular we deduce: if $p \geqq 2,|f(x)| \leqq \pi /(2 p+\delta)$, and

$$
\Phi(x)=\frac{1}{\pi} \int \frac{1+u x}{u-x} \frac{f(u)}{1+u^{2}} d u+i f(x),
$$

then $\exp \{\Phi(x)\} \in \mathbb{R}_{p}^{+}$. Setting $f(x)=\alpha \pi\left\{\chi_{E}(x)-1 / 2\right\}$ we have that $\rho \in \mathbb{R}_{p}^{+}$if $p \geqq 2$.

For $p$ an even integer we deduce from Lemma 4 that also $\rho \in W_{p \text {. }}$ To prove this for general $p \geqq 2$, find even integers $p_{1}, p_{2}$ with $p_{1} \leqq p \leqq p_{2}, p^{-1}$ $=(1-t) p_{1}^{-1}+t p_{2}^{-1}$, and set $\rho_{1}=\rho^{p / p_{1}}, \rho_{2}=\rho^{p / p_{2}}$. Then by what has already been shown $\rho_{1} \in W_{p_{1}}$ and $\rho_{2} \in W_{p_{2}}$, and it follows from Stein's theorem that $\rho \in W_{p}$.

For $p \leqq 2$ we can use Lemma 1 and what has already been shown to deduce that $\rho \in W_{p .}$. Moreover it then follows from Lemma 2 that $\rho \in \mathbb{R}_{p}$. Now $\rho(x)$ $=\exp \{\Phi(x)\}$ where $\Phi$ is given by (16) for a particular bounded function $f$. Moreover $\Phi(x)$ is the boundary function of $\Phi(z)$ given by (14). Thus it suffices to show the following: If $f$ is real and bounded and

$$
\int_{-\infty}^{\infty} \frac{\left|e^{\Phi(x)}\right|}{1+|x|^{p}} d x \leqq A,
$$

then

$$
\int_{-\infty}^{\infty} \frac{\left|e^{\Phi(x+i y)}\right|}{1+|x|^{p}} d x \leqq A,
$$

when $1<p \leqq 2$. (Note that we have replaced $p \Phi$ by $\Phi$, a matter of no importance. Also the two constants denoted by " $A$ " may be different.)

We begin by showing that

$$
\frac{2}{\pi} \int \frac{1+u z}{u-z} \frac{f_{\mu}(u)}{1+u^{2}} d u=0, \quad g z>0 .
$$


This is clear if $f$ vanishes outside a finite interval since then $f_{\mu} \in \mathbb{R}_{\tau}-$ for any $r>1$ and we may use an argument along the lines of that used above to show that $f \in R_{p}^{+}$implied $f_{\pi}=f$. For general $f$ let

$$
f^{(n)}(u)= \begin{cases}f(u), & |u|<n, \\ 0, & |u| \geqq n .\end{cases}
$$

Since

$$
\int \frac{\left|f_{\mu}^{(n)}(u)-f_{\mu}(u)\right|^{r}}{1+u^{2}} d u \rightarrow 0
$$

as $n \rightarrow \infty$ (this follows from the classical M. Riesz theorem on conjugate functions if we use the change of variable (15)), and since (18) holds for each $f^{(n)}$, we deduce (18) for $f$ itself.

Set $z=x+i y$ in (18) and take the real part of both sides. We obtain

$$
\frac{1}{\pi} \int\left\{\frac{u-x}{(u-x)^{2}+y^{2}}-\frac{u}{1+u^{2}}\right\} f(u) d u=\frac{y}{\pi} \int \frac{R \Phi(u)}{(u-x)^{2}+y^{2}} d u .
$$

Therefore

$$
\begin{aligned}
\left|e^{\Phi(x+i y)}\right| & =\exp \left(\frac{1}{\pi} \int\left\{\frac{u-x}{(u-x)^{2}+y^{2}}-\frac{u}{1+u^{2}}\right\} f(u) d u\right) \\
& =\exp \left(\frac{y}{\pi} \int \frac{R \Phi(u)}{(u-x)^{2}+y^{2}} d u\right) .
\end{aligned}
$$

Applying Jensen's inequality to the last integral, we obtain

$$
|\exp \Phi(x+i y)| \leqq \frac{y}{\pi} \int \frac{|\exp \Phi(u)|}{(u-x)^{2}+y^{2}} d u
$$

and so

$$
\begin{aligned}
\int \frac{|\exp \Phi(x+i y)|}{1+|x|^{p}} & d x \\
& \leqq \frac{1}{\pi} \int|\exp \Phi(u)| d u \int \frac{y d x}{\left\{(u-x)^{2}+y^{2}\right\}\left\{1+|x|^{p}\right\}}
\end{aligned}
$$

Now by a straightforward computation the inner integral on the right is seen to be at most $A /\left(1+|u|^{p}\right)$ if $p \leqq 2$. Thus (17) is proved and the proof if Lemma 5 is complete.

The following lemma is the analogue for Hilbert transforms of a result of Hirschman [4, Theorem 2.1] on conjugate functions. Our result can be derived very easily from Theorem 319 of [2] but for the sake of continuity we prefer to make use of what has gone before. 
Lemma 6. If $-p^{-1}<\alpha<q^{-1}$ then $|x|^{\alpha} \in W_{p}$.

Proof. Set

$$
\rho(x)= \begin{cases}x^{\alpha}, & x>0 \\ |x|^{\alpha} \exp i \pi \alpha, & x<0 .\end{cases}
$$

Then since $\rho(x)$ is the boundary function of $z^{\alpha}$ (determined in $g z>0$ by $0<\arg z<\pi$ ) it belongs to $\mathfrak{R}_{p}^{+}$. Consequently the result follows from Lemma 4 if $p$ is an even integer and $\alpha p$ is not an odd integer. If $\alpha p$ is odd then for sufficiently small $\epsilon$ we shall have $-p^{-1}<\alpha \pm \epsilon<q^{-1}$ and neither of $(\alpha \pm \epsilon) p$ will be odd. Therefore the functions $|x|^{\alpha \pm e}$ belong to $W_{p}$, so by Stein's theorem $|x|^{\alpha} \in W_{p}$. For general $p \geqq 2$ find even integers $p_{1}, p_{2}$ satisfying $p_{1} \leqq p \leqq p_{2}$. Then if $\alpha \leqq 0$ we take, in our application of Stein's theorem, $k_{1}=u_{1}=|x| \alpha_{p} / p_{1}$ and $k_{2}=u_{2}=|x|_{\alpha p / p_{2}}$, and the result follows. If $\alpha \geqq 0$ we take $k_{1}=u_{1}=|x|^{\alpha q / q_{1}}$ and $k_{2}=u_{2}=|x|_{\alpha q / z_{2}}$, and the result follows. This takes care of all $p \geqq 2$; the result for $p \leqq 2$ now follows from Lemma 1 .

Given two functions $\rho_{1}$ and $\rho_{2}$, we shall say that they are of the same size on $E$ if both $\rho_{1} / \rho_{2}$ and $\rho_{2} / \rho_{1}$ are bounded there.

LEMma 7. If $\rho_{1}$ and $\rho_{2}$ are of the same size on $E \cup F$ and $\rho_{1} \in W_{p, E, F}$ then also $\rho_{2} \in W_{p, E, \boldsymbol{F} \text {. }}$

Proof. Clear.

Lemma 8. Let $E_{i}(i=1, \cdots, n)$ be disjoint sets with union $(-\infty, \infty)$. If $\rho \in W_{p, E_{i}, E_{j}}$ for each pair $i, j$ then $\rho \in W_{p}$.

Proof. Assume we have

$$
\int_{E_{j}}|\rho(x) \tilde{g}(x)|^{p} d x \leqq A_{i j}^{p} \int_{E_{i}}|\rho(x) g(x)|^{p} d x
$$

for every $g$ vanishing outside $E_{i}$ and such that $g(x) /(1+|x|) \in L_{1}$. Assume $f(x) /(1+|x|) \in L_{1}$ and write $f=f_{1}+\cdots+f_{n}$ where $f_{i}=0$ outside $E_{i}$. Then

$$
\begin{aligned}
\int|\rho(x) \tilde{f}(x)|^{p} d x & =\sum_{j} \int_{E_{j}}|\rho(x) \tilde{f}(x)|^{p} d x \\
& \leqq \sum_{j}\left\{\sum_{i}\left[\int_{E_{j}}\left|\rho(x) \tilde{f}_{i}(x)\right|^{p} d x\right]^{1 / p}\right\}^{p} \\
& \leqq \sum_{j}\left\{\sum_{i} A_{i j}\right\}^{p} \cdot \int|\rho(x) f(x)|^{p} d x .
\end{aligned}
$$

Lemma 9. Assume $-p^{-1}<\alpha_{k}<q^{-1} \quad(k=1, \cdots, n), \quad-p^{-1}<\beta+\sum_{1}^{n} \alpha_{k}$ $<q^{-1}, x_{1}, \cdots, x_{n}$ real and distinct, and set 


$$
\rho(x)=\left(1+|x|^{\beta}\right) \prod_{k=1}^{n}\left|x-x_{k}\right|^{\alpha_{k}} .
$$

Then $\rho$ belongs to $W_{p}$.

Proof. Assuming that $x_{1}<\cdots<x_{n}$, find $a_{0}, \cdots, a_{n}$ so that

$$
a_{0}<x_{1}<a_{1}<x_{2}<\cdots<x_{n}<a_{n} .
$$

Let $I_{k}=\left(a_{k-1}, a_{k}\right)$, and split $I_{k}$ into subintervals $I_{k}^{-1}, I_{k}^{0}, I_{k}^{1}$ so that $x_{k}$ is in the interior of $I_{k}^{0}$. Finally let $I_{\infty}^{-1}=\left(a_{n}+1, \infty\right), I_{\infty}^{1}=\left(-\infty, a_{0}-1\right)$ and $I_{\infty}^{0}=\left(a_{0}-1, a_{0}\right)$ $\cup\left(a_{n}, a_{n}+1\right)$. We shall show that for each pair of sets

$$
I_{r}^{i}, I_{s}^{j}(r, s=1, \cdots, n, \infty ; i, j=-1,0,1)
$$

$\rho$ belongs to $W_{p, I_{r}, I_{j} j}$. We consider three cases.

(i) Sets not adjacent. The result follows from Lemma 3.

(ii) Sets adjacent but $r \neq s$. Then on $I_{r}^{t} \cup I_{s}^{j} \rho$ is of the same size as 1 and the result follows from Lemma 7.

(iii) Sets adjacent and $r=s$. If $r=s=\infty$ then on $I_{r}^{t} \cup I_{a}^{s} \rho(x)$ is of the same size as $|x|^{\beta+\Sigma \alpha_{k}}$ and the result follows from Lemmas 7 and 6. If $r=s \neq \infty$ then on $I_{r}^{t} \cup I_{s}^{j} \rho(x)$ is of the same size as $\left|x-x_{r}\right| \alpha_{r}$ and again the result follows from Lemmas 7 and 6 , if we note that any translate of a function in $W_{p}$ also belongs to $W_{p}$.

3. The Riemann-Hilbert problem in $L_{p}$. This can be stated as follows: Given $G(x)$ defined on $(-\infty, \infty)$ and $g(x) \in L_{p}(-\infty, \infty)$, find functions $f_{+}$ and $f_{-}$belonging to $L_{p}^{+}$and $L_{p}^{-}$respectively such that

$$
G(x) f_{+}(x)=f_{-}(x)+g(x)
$$

almost everywhere. Throughout this section we shall assume that $G(x)$ and $1 / G(x)$ are bounded, and in the sequel we always take $p>1$.

Theorem I. Assume $G(x)=G^{+}(x) G^{-}(x)$ where $G^{+} \in \mathbb{R}_{q}^{+}$and $1 / G^{-} \in \mathbb{R}_{a}^{-}$. A necessary and sufficient condition that the Riemann-Hilbert problem (20) have a unique solution $\left(f_{+}, f_{-}\right) \in L_{p}^{+} \times L_{p}^{-}$for each $g \in L_{p}$ is that $G^{-} \in W_{p}$. If this condition is met the solution is given by

$$
f_{+}=\frac{1}{G^{+}}\left(\frac{g}{G^{-}}\right)_{+}, \quad f_{-}=-G^{-}\left(\frac{g}{G^{-}}\right)_{-} .
$$

Proof. Suppose $g \in L_{p}$ has the property that also $g / G-\in L_{p}$, and that for this $g$ equation (20) has a solution. Then

$$
G^{+} f_{+}=\frac{f_{-}}{G^{-}}+\frac{g}{G^{-}} .
$$

Since the three functions in (22) belong to $\Omega_{1}$ we can apply the operator $\pi$ to both sides. Since $G^{+} f_{+} \in R_{1}^{+}$we have $\left(G^{+} f_{+}\right)_{x}=G^{+} f_{+}$, and since $f_{-} / G^{-} \in R_{1}^{-}$we 
have $\left(f_{-} / G^{-}\right)_{\pi}=0$. Also, since $g / G^{-} \in L_{p}$ we have $\left(g / G^{-}\right)_{\pi}=\left(g / G^{-}\right)_{+}+$const. Therefore

$$
G^{+} f_{+}=\left(\frac{g}{G^{-}}\right)_{+}+\text {const. }
$$

Since $G^{+} f_{+} \in R_{1}$ and $\left(g / G^{-}\right)_{+} \in L_{p}$ the constant must be zero. Thus we obtain the first statement of (21); the second follows similarly from (22) by applying $\mu$ to both sides.

Assume now that for every $g \in L_{p}$ (20) has a unique solution, and define the operator $G$ on $L_{p}^{+}$by

$$
G f=(G f)_{+}, \quad f \in L_{p}^{+} .
$$

Then for $g \in L_{p}^{+}$the equation $G f=g$ will have a unique solution in $L_{p}^{+}$, namely the function $f_{+}$in the solution of (20). Now since $G(x)$ is bounded, $G$ is a bounded operator on the Banach space $L_{p}^{+}$. (That $L_{p}^{+}$is a Banach space was proved in \$2.) Therefore by one version of the closed graph theorem [7, $\$ 7 \mathrm{G}], G^{-1}$ is a bounded operator. Take a function $g \in L_{p}$ so that also $g / G-\in L_{p}$. Then if $f_{+}$is the function in (20) we have $\left(G f_{+}\right)_{+}=g_{+}$, i.e., $G f_{+}=g_{+}$, and so $f_{+}=G^{-1} g_{+}$. It follows therefore that

$$
\left\|f_{+}\right\|_{p} \leqq\left\|G^{-1}\right\|\left\|g_{+}\right\|_{p} \leqq A\|g\|_{p},
$$

where $A$ is independent of $g$. Since $f_{+}$is given by (21), we have

$$
\left\|\frac{1}{G^{+}}\left(\frac{g}{G^{-}}\right)\right\|_{p} \leqq A\left\|_{g}\right\|_{p}
$$

and so, since $1 / G^{+}=G^{-} / G$ is of the same size as $G^{-}$(since $1 / G$ is bounded), we have

$$
\left\|G^{-}\left(\frac{g}{G^{-}}\right)^{\sim}\right\|_{p}=\left\|2 i G^{-}\left(\frac{g}{G^{-}}\right)_{+}-i g\right\|_{p} \leqq A\left\|_{g}\right\|_{p:}
$$

Replacing $g / G^{-}$by $g$, we have

$$
\left\|G^{-} g\right\|_{p} \leqq A\left\|G^{-} g\right\|_{p}
$$

whenever $g \in L_{p}$ and $G^{-} g \in L_{p}$. This implies $G^{-} \in W_{p}$.

To prove the sufficiency of the condition, we assume $G^{-} \in W_{p}$. The uniqueness has already been proved since we showed above, in particular, that the only solution of (20) with $g=0$ is given by $(21)$, i.e. $f_{+}=f_{-}=0$. It remains therefore to show that $(21)$ is indeed a solution of (20). Note that since $g \in L_{p}$ and $1 / G^{-} \in \mathfrak{R}_{q}$ we have $g / G^{-} \in \mathfrak{R}_{1}$, so $\left(g / G^{-}\right)_{ \pm}$make sense. Also $\left(g / G^{-}\right)_{+}$ $+\left(g / G^{-}\right)_{-}=g / G^{-}$, from which (20) follows. We must still show that $f_{+} \in L_{p}^{+}$ and $f_{-} \in L_{p}^{-}$. Assume first that $g / G^{-} \in L_{p}$. Then $f_{+} \in R_{1}^{+}$and by (23) (which follows from (24) just as (24) follows from it) $f_{+} \in L_{p}$. Write $f_{+}=f^{+}+f^{-}$with 
$f^{+} \in L_{p}^{+}$and $f^{-} \in L_{p}^{-}$, and apply $\mu$ to both sides. We obtain $0=$ const $+f^{-}$, from which $f^{-}=0$, and so $f_{+}=f^{+} \in L_{p}^{+}$. For a general $g \in L_{p}$ write

$$
g=\underset{n \rightarrow \infty}{\operatorname{lip} . \mathrm{m}} . g^{(n)}
$$

with each $g^{(n)} / G^{-} \in L_{p}$ and define $f_{+}^{(n)}$ from $g^{(n)}$ by (21). Then from (23) we see that

$$
f_{+}=\underset{n \rightarrow \infty}{\left.\operatorname{lp} . i_{n}\right)} f_{+}^{(n)}
$$

and we know that $f_{+}^{(n)} \in L_{p}^{+}$. Therefore $f_{+} \in L_{p}^{+}$. A similar argument shows that $f_{-} \in L_{p}^{-}$, and the proof of the theorem is complete.

It will be convenient on occasion to consider, instead of (20), the equation

$$
G f_{+}=g_{+}
$$

where $g_{+} \in L_{p}^{+}$is given and $f_{+} \in L_{p}^{+}$sought. This problem is completely equivalent to (20); for if (20) can be solved with $g=g_{+}$then the function $f_{+}$ satisfies (25); conversely given $g \in L_{p}$ if (25) can be solved for $f_{+}$then the pair $\left(f_{+},\left(G f_{+}\right)_{-}-g_{-}\right)$is a solution of $(20)$. In particular the unique solvability of (20) for each $g \in L_{p}$ is equivalent to the invertibility of $G$.

Using the results of $\S 2$, the equations (20) and (25) can be investigated for a rather large class of functions $G(x)$. Throughout the remainder of this section we shall assume

$1^{\circ}$ there may be defined an arg $G(x)$ which is continuous except for jumps at $x_{1}, \cdots, x_{n}$; the limits arg $G( \pm \infty)$ exist. We set

$$
\begin{array}{ll}
a_{k}=\arg G\left(x_{k}+\right)-\arg G\left(x_{k}-\right), & 1 \leqq k \leqq n, \\
a_{0}=\arg G(-\infty)-\arg G(+\infty) . &
\end{array}
$$

If $1(x)$ denotes the characteristic function of $(0, \infty)$, the function

$$
\gamma(x)=\arg G(x)-\sum_{k=1}^{n} a_{k} 1\left(x-x_{k}\right)+\frac{1}{\pi} \sum_{k=0}^{n} a_{k} \tan ^{-1} x
$$

is continuous and has equal limits at $\pm \infty$. We assume also

$2^{\circ}$ the function

$$
\int \frac{1+y x}{y-x} \frac{\gamma(y)}{1+y^{2}} d y
$$

is bounded.

It follows from $2^{\circ}$ using the inequality (19) that $\exp \left\{i \gamma_{\pi}(x)\right\}$ is the boundary function of a function analytic, bounded, and bounded away from zero in $9 z>0$; similarly $\exp \left\{i \gamma_{\mu}(x)\right\}$ is the boundary function of a function analytic, bounded, and bounded away from zero in $g z<0$. Moreover 


$$
\exp i \gamma(x)=\exp i \gamma_{\pi}(x) \exp i \gamma_{\mu}(x) \text {. }
$$

The condition $2^{\circ}$ is easily seen to be satisfied if the functions $\gamma(x)$ and $\gamma\left(x^{-1}\right)$ satisfy a Lipschitz condition.

TheOREM II. For $k=1, \cdots, n$ write $a_{k}=b_{k}+c_{k}$ where $b_{k}$ is an integral multiple of $2 \pi$ and $-2 \pi p^{-1}<c_{k} \leqq 2 \pi q^{-1}$; write $a_{0}=b_{0}+c_{0}$ where $b_{0}$ is an integral multiple of $2 \pi$ and $-2 \pi p^{-1}<-c_{0} \leqq 2 \pi q^{-1}$.

(a) A ssume no $c_{k}(k=1, \cdots, n)$ equals $2 \pi q^{-1},-c_{0} \neq 2 \pi q^{-1}$. We set

$$
m=\frac{1}{2 \pi} \sum_{k=0}^{n} b_{k}
$$

Then

(i) if $m=0 G$ is invertible,

(ii) if $m>0 G$ is onto with null space of dimension $m$,

(iii) if $m<0 G$ is one-one with range of deficiency $-m$.

(b) If any $c_{k}(k=1, \cdots, n)$ equals $2 \pi q^{-1}$ or if $-c_{0}=2 \pi q^{-1}$ then $G$ is not invertible.

Proof. (a) Since $G$ is bounded and bounded away from zero, $\log |G|$ is bounded. Now $(\log |G|)_{\pi}$ is the boundary function of

$$
\frac{1}{2 \pi i} \int \frac{1+u z}{u-z} \frac{\log |G(u)|}{1+u^{2}} d u \quad z=x+i y, y>0
$$

which has real part

$$
\frac{y}{2 \pi} \int \frac{\log |G(u)|}{(u-x)^{2}+y^{2}} d u,
$$

and this is bounded. It follows that $\exp \left\{(\log |G|)_{\pi}\right\}$ is the boundary function of a function analytic, bounded, and bounded away from zero in $g z>0$. Similarly $\exp \left\{(\log |G|)_{\mu}\right\}$ is the boundary function of a function analytic, bounded, and bounded away from zero in $g z<0$. Moreover

$$
|G|=\exp (\log |G|)_{\pi} \exp (\log |G|)_{\mu} .
$$

Now we have

$$
\begin{aligned}
\operatorname{sgn} G(x)= & \exp \left(i\left\{\gamma(x)+\left[\sum_{k=1}^{n} c_{k} 1\left(x-x_{k}\right)\right]-\frac{1}{\pi}\left[\sum_{k=0}^{n} c_{k} \tan ^{-1} x\right]\right\}\right. \\
& \left.-2 i m \tan ^{-1} x\right) \\
= & \left(\frac{i+x}{i-x}\right)^{m} \exp i \gamma(x) \exp i\left\{\left[\sum_{k=1}^{n} c_{k} 1\left(x-x_{k}\right)\right]-\frac{1}{\pi}\left[\sum_{k=0}^{n} c_{k} \tan ^{-1} x\right]\right\},
\end{aligned}
$$


and we still need a factorization for the third term. This is straightforward. In fact if we set

$$
\begin{array}{ll}
\rho_{+}(z)=(i+z)^{(1 / 2 \pi) \Sigma_{k-0}^{n} c_{k}} \prod_{k=0}^{n}\left(z-x_{k}\right)^{-c_{k} / 2 \pi}, & g z>0, \\
\rho_{-}(z)=(i-z)^{(-1 / 2 \pi) \Sigma_{k-0}^{n} c_{k}} \prod_{k=1}^{n}\left(z-x_{k}\right)^{c_{k} / 2 \pi}, & g z<0,
\end{array}
$$

(where the arguments are determined by $0<\arg \left(z-x_{k}\right)<\pi, 0<\arg (i+z)<\pi$ for $g z>0$ and $\pi<\arg \left(z-x_{k}\right)<2 \pi, 0<\arg (i-z)<\pi$ for $\left.g z<0\right)$ with corresponding boundary functions $\rho_{+}(x)$ and $\rho_{-}(x)$ then

$$
\rho_{+}(x) \rho_{-}(x)=\exp i\left\{\left[\sum_{k=1}^{n} c_{k} 1\left(x-x_{k}\right)\right]-\frac{1}{\pi}\left[\sum_{k=0}^{n} c_{k} \tan ^{-1} x\right]\right\} .
$$

Finally let us set

$$
\begin{aligned}
G^{+}(x) & =\rho_{+}(x) \exp (\log |G|)_{\pi}(x) \exp i \gamma_{\pi}(x), \\
G^{-}(x) & =\rho_{-}(x) \exp (\log |G|)_{\mu}(x) \exp i \gamma_{\mu}(x), \\
H(x) & =\left(\frac{i+x}{i-x}\right)^{m}, G_{1}(x)=G^{+}(x) G^{-}(x) .
\end{aligned}
$$

Since $G^{+} \in \mathbb{R}_{q}^{+}$and $1 / G^{-} \in \mathbb{R}_{q}^{-}$and since, by Lemmas 9 and $7, G^{-}$belongs to $W_{p}$, we know from Theorem I that the operator on $L_{p}^{+}$

$$
G_{1}: f \rightarrow\left(G_{1} f\right)_{+}
$$

is invertible. If $m=0$ then $G$ is $G_{1}$ and is therefore invertible. Otherwise we must consider the operator on $L_{p}^{+}$

$$
H: f \rightarrow(H f)_{+} \text {. }
$$

Assume first that $m>0$. Then

$$
\left(\frac{i-z}{i+z}\right)^{m}
$$

is analytic and bounded in $g z>0$. It follows that for $g \in L_{p}^{+}$the equation $\boldsymbol{H} \boldsymbol{f}=\boldsymbol{g}$ always has the solution

$$
f(x)=\left(\frac{i-x}{i+x}\right)^{m} g(x),
$$

so $H$ is onto. To find the general solution of the homogeneous equation, note that if $f_{+} \in L_{p}^{+}$and $H f_{+}=0$ then $H f_{+}=g_{-}$where $g_{-} \in L_{p}^{-}$. Then

$$
(i+x)^{m} f_{+}(x)=(i-x)^{m} g_{-}(x),
$$

and we can apply Carleman's theorem and the inequality (14) to deduce that both sides must be $P(x)$, a polynomial of degree less than $m$. That in fact 


$$
f_{+}(x)=\frac{P(x)}{(i+x)^{m}}
$$

does satisfy $H f_{+}=0$ for any such $P(x)$ is immediate since then $H f_{+}$ $=P(x)(i-x)^{-m} \in L_{p}^{-}$.

We consider now the case $m<0$. That $H$ is one-one with range of deficiency $-m$ could be proved from the above by considering adjoints. However it is also easy to proceed directly. Assume that for a given $g_{+} \in L_{p}^{+}$the equation $H f_{+}=g_{+}$has a solution $f_{+} \in L_{p}^{+}$. Then $H f_{+}=g_{+}+g_{-}$with $g_{-} \in L_{p}^{-}$, and we have

$$
\frac{(i-x)^{m-k}}{(i+x)^{-m}} f_{+}(x)=\frac{g_{+}(x)}{(i-x)^{k}}+\frac{g_{-}(x)}{(i-x)^{k}}, \quad 0<k \leqq-m .
$$

Integrating from $-\infty$ to $\infty$, the first and last terms in (26) give zero. This follows easily from the fact $\left[3\right.$, Lemma 2.4] that for $h(z) \in \mathfrak{W}_{p}, h(z) \rightarrow 0$ uniformly as $z \rightarrow \infty$ in any half-plane $g z \geqq \delta>0$. Hence we obtain

$$
\int_{-\infty}^{\infty} \frac{g_{+}(x)}{(i-x)^{k}} d x=0, \quad 0<k \leqq-m,
$$

so the range of $\boldsymbol{H}$ has deficiency at least $-m$. But the conditions (27) are sufficient for the solvability of $\boldsymbol{H} f_{+}=g_{+}$. In fact we simply take

$$
f_{+}(x)=\left(\frac{i-x}{i+x}\right)^{m} g_{+}(x) \text {. }
$$

That $f_{+} \in L_{p}^{+}$follows from the fact that $g_{+}(z)$, the $\mathfrak{W}_{p}$ function whose boundary function is $g_{+}(x)$, has, by (27), a zero at $z=i$ of multiplicity at least $-m$.

We have shown that if $m>0$ then $H$ is onto with null space of dimension $m$, and if $m<0$ then $H$ is one-one with range of deficiency $-m$. Moreover $G_{1}$ is invertible, and $G=G_{1} M$ for $m<0$ and $G=M G_{1}$ for $m>0$. This completes the proof of part (a).

(b) To prove that if any $c_{k}=2 \pi q^{-1}(k=1, \cdots, n)$ or if $-c_{0}=2 \pi q^{-1}$ then $G$ is not invertible, we shall show that $G$ is the uniform limit of noninvertible operators. (The invertible elements of a Banach algebra form an open set $[7, \S 22 \mathrm{C}]$.) Define

$$
G_{\epsilon}(x)=G(x) \exp i\left\{\sum \epsilon_{k} 1\left(x-x_{k}\right)+(1 / \pi)\left(\epsilon_{0}-\sum \epsilon_{k}\right) \tan ^{-1} x\right\}
$$

where $\epsilon_{k}$ is small and nonzero if $c_{k}=2 \pi q^{-1}$ and zero otherwise for $k=1, \cdots, n$, and $\epsilon_{0}$ is small and nonzero if $-c_{0}=2 \pi q^{-1}$ and zero otherwise. Then $G_{\epsilon}$ satisfies the conditions of part (a) and so has associated with it a number $m_{\mathrm{e}}$ which depends of course on the particular choice of the $\epsilon_{i}$. It is clear that by reversing the sign of one $\epsilon_{i}$ the associated $m_{\epsilon}$ is altered by \pm 1 . Therefore by an appropriate choice of the $\epsilon_{i}$ we can guarantee that $m_{\epsilon} \neq 0$, i.e. $G_{\epsilon}$ is not invertible. Since $\left\|G_{.}-G\right\|$ can be made as small as desired (note that $\left\|G_{\mathrm{e}}-G\right\|$ $\leqq A$ sup $\left.\left|G_{\bullet}(x)-G(x)\right|\right)$, $G$ is not invertible. 
4. The equation $K_{0} f=g$. This is

$$
K_{0} f(x) \equiv A(x) f(x)+\frac{B(x)}{\pi i} \int_{E} \frac{f(y)}{y-x} d y=g(x), \quad x \in E,
$$

where $g \in L_{p}(E)$ is given and $f \in L_{p}(E)$ is sought. We assume throughout this section that $A(x)$ and $B(x)$ are bounded, and that $A(x)^{2}-B(x)^{2}$ is bounded away from zero.

Assume (28) holds, and extend $f$ and $g$ to all of $(-\infty, \infty)$ by defining them to be zero outside $E$. Set

$$
F^{+}(x)=f_{+}(x), \quad F^{-}(x)=-f_{-}(x) .
$$

Then we have

$$
\begin{aligned}
& F^{+}(x)-F^{-}(x)=f(x), \\
& F^{+}(x)+F^{-}(x)=\frac{1}{\pi i} \int_{E} \frac{f(y)}{y-x} d y
\end{aligned}
$$

for (almost) all $x$. Therefore (28) implies

$$
\{A(x)+B(x)\} F^{+}(x)-\{A(x)-B(x)\} F^{-}(x)=g(x), \quad x \in E .
$$

Define

$$
G(x)=\left\{\begin{array}{cl}
\frac{A(x)+B(x)}{A(x)-B(x)}, & x \in E, \\
1, & x \in E
\end{array}\right.
$$

and

$$
g_{1}(x)=\frac{g(x)}{A(x)-B(x)} .
$$

(Note that by our assumptions on $A$ and $B, G(x)$ is bounded and bounded away from zero; and $g \in L_{p}$ if and only if $g_{1} \in L_{p}$.) Then (32) gives

$$
G(x) F^{+}(x)=F^{-}(x)+g_{1}(x),
$$

at least for $x \in E$. But for $x \notin E$ (34) is the statement $F^{+}(x)=F^{-}(x)$, i.e., $f_{+}(x)+f_{-}(x)=0$, which is true since $f(x)=0$ outside $E$. Thus (28) implies (34), where $F^{+}$and $F^{-}$are defined by (29). Conversely suppose we could solve (34) for $\left(F^{+}, F^{-}\right) \in L_{p}^{+} \times L_{p}^{-}$. Then we define $f(x)$ by (30). Since $F^{+}-F^{-}=f$ $=f_{+}+f_{-}$we deduce (29) and so also (31). Therefore (34) gives (28).

We have demonstrated the equivalence of equations of the form (28) with Riemann-Hilbert problems (34) where $g_{1}$ vanishes outside $E$. Thus with very little work we can establish the analogue for the operator $\boldsymbol{K}_{0}$ of Theorem II above. 
Theorem III. Suppose $G(x)$ satisfies the conditions $1^{\circ}$ and $2^{\circ}$ of $\$ 3$, and let $a_{k}, b_{k}$, and $c_{k}$ be as defined there.

(a) Assume no $c_{k}(k=1, \cdots, n)$ equals $2 \pi q^{-1},-c_{0} \neq 2 \pi q^{-1}$. Set

$$
m=\frac{1}{2 \pi} \sum_{k=0}^{n} b_{k} .
$$

Then

(i) if $m=0 K_{0}$ is invertible,

(ii) if $m>0 K_{0}$ is onto with null space of dimension $m$,

(iii) if $m<0 K_{0}$ is one-one with range of deficiency $-m$.

(b) If any $c_{k}(k=1, \cdots, n)$ equals $2 \pi q^{-1}$ or if $-c_{0}=2 \pi q^{-1}$ then $K_{0}$ is not invertible.

Proof. Part (a, i) follows immediately from Theorem II. As for (a, ii) we see that $K_{0} f=0$ is equivalent to $G F^{+}=0$, where $F^{+}$is given by (29). Therefore the result will follow from Theorem II if we can show that in this case $f=0$ is equivalent to $F^{+}=0$ (i.e., the number of linearly independent $f^{\prime}$ s equals the number of linearly independent $F^{+}$'s). That $f=0$ implies $F^{+}=0$ is clear. If $F^{+}=0$ then (34), with $g_{1}=0$ gives $F^{-}=0$, and so $f=F^{+}-F^{-}=0$.

Before considering ( $a$, iii) we digress for a moment, and notice that the dual space of $L_{p}^{+}$is $L_{q}^{+}$. For if $\mathcal{L}$ is a continuous linear functional on $L_{p}^{+}$it can be extended, by the Hahn-Banach theorem, to a continuous linear functional on $L_{p}$. Thus for some $g \in L_{q}$ we have $\& f=(f, g)=\left(f, g_{+}\right)+\left(f, g_{-}\right)$. Now

$$
\left(f, g_{-}\right)=\int f(x)\left[g_{-}(x)\right]^{*} d x
$$

and $f \bar{g}_{-} \in L_{1}^{+}$. This last statement implies that both $f \bar{g}_{-}$and $\left(f \bar{g}_{-}\right) \sim$ belong to $L_{1}$, and so by a theorem of Kober [6, Theorem $\left.1(\mathrm{a})\right]$ we have $\int f \bar{g}-d x=0$. Thus $\mathscr{L} f=\left(f, g_{+}\right)$. Another fact we shall need is that a function $f$ in $L_{p}^{+}$which is not identically zero can vanish only on a set of measure zero. In fact it is known [10] that if $g(\theta)$ is the boundary function of a nonzero function in $H_{p}$ of the unit circle, then $\log |g| \in L_{1}$. If we use the change of variable $x=\tan \theta / 2$ we can conclude that

$$
\int_{-\infty}^{\infty} \frac{|\log | f(x)||}{1+x^{2}} d x<\infty
$$

and so $f$ may vanish only on a set of measure zero.

Returning to the proof of (a, iii), we assume $m<0$ and denote by $R$ the range of $K_{0}$ in $L_{p}(E)$ and by $R_{+}$the range of $G$ in $L_{p}^{+}$. By Theorem II $R_{+}$ has deficiency $-m$. Since the dual of $L_{p}^{+}$is $L_{q}^{+}$this means we can find $-m$ linearly independent functions $h_{1}, \cdots, h_{-m}$ belonging to $L_{q}^{+}$and orthogonal to $R_{+}$. Each $h_{k}$ is orthogonal to any $g_{1} \in L_{p}$ for which (34) has a solution. 
For $h_{k}$ is orthogonal to $\left(g_{1}\right)_{+}$(the latter function belonging to $R_{+}$) and to $\left(g_{1}\right)_{-}$(by Kober's theorem, since $h_{k}\left\{\left[\left(g_{1}\right)_{-}\right]^{*} \in L_{1}^{+}\right)$. Therefore each of the functions

$$
\frac{h_{k}(x)}{[A(x)]^{*}-[B(x)]^{*}}
$$

is orthogonal to $R$. Moreover since any linear combination of the $h_{k}$ belongs to $L_{q}^{+}$this linear combination cannot vanish on $E$ without vanishing identically, i.e., the $h_{k}$ are linearly independent on $E$. Thus we have found $-m$ linearly independent functions orthogonal to $R$, and $R$ has deficiency at least $-m$.

Next assume $h$ is orthogonal to $R$, and extend $h$ to be zero outside $E$. Then for any $f \in L_{p}(E), f$ also being defined to be zero outside $E$, we have

$$
0=(h, A f-i B \bar{f})=(\bar{A} h-i(\bar{B} h) \sim, f)
$$

and so

$$
[A(x)]^{*} h(x)+\frac{1}{\pi i} \int_{E} \frac{[B(y)]^{*} h(y)}{y-x} d y=0, \quad x \in E .
$$

Set

$$
k(x)=B(x)[h(x)]^{*} .
$$

Then multiplying (35) by $[B(x)]^{*}$ and taking complex conjugates we obtain

$$
A(x) k(x)-\frac{B(x)}{\pi i} \int_{E} \frac{k(y)}{y-x} d y=0, \quad x \in E .
$$

Now $h \not \equiv 0$ implies $k \not \equiv 0$. For assume $k \equiv 0$ and let $S$ be the set on which $B$ vanishes. Then by (36) $h$ vanishes on the complement of $S$ and by (35) $\bar{A} h$ vanishes everywhere. Since $\bar{A} \neq 0$ on $S$ (recall that $A^{2}-B^{2} \neq 0$ ) we have $h=0$ on $S$. Therefore $h \equiv 0$, which is a contradiction. From what we have shown it follows that the deficiency of $R$ is at most the dimension in $L_{q}(E)$ of the set of solutions of (37). Now the " $G$ " for equation (37) is just $1 / G$ and so (since we are considering the operator on $L_{q}$ ) the " $m$ " is $-m$. But by part (a, ii) the set of solutions of (37) has dimension $-m$, so $R$ has deficiency at most $-m$.

(b) This follows from part (a) just as part (b) of Theorem II followed from the corresponding part (a). We omit the details.

5. The case $A(x)=-\lambda, B(x)=1$. The equation is then

$$
\frac{1}{\pi i} \int_{E} \frac{f(y)}{y-x} d y=\lambda f(x)+g(x) .
$$

Defining the operator $T$ by 


$$
T f(x)=\frac{1}{\pi i} \int_{E} \frac{f(y)}{y-x} d y
$$

the equation can then be written simply as $T f=\lambda f+g$. The relevant function $G$ is given by

$$
G(x)=\left\{\begin{array}{cl}
\frac{\lambda-1}{\lambda+1}, & x \in E, \\
1, & x \notin E .
\end{array}\right.
$$

The cases $\lambda= \pm 1$ are special since then either $G$ or $1 / G$ is unbounded. However they are also simple cases since the points \pm 1 belong to the point spectrum of $T$ if $E=(-\infty, \infty)$ and to the continuous spectrum otherwise. Taking the case $\lambda=1$ ( $\lambda=-1$ being entirely analogous), (32) is equivalent to

$$
\begin{aligned}
F^{-}(x) & =-\frac{1}{2} g(x), & x & \in E, \\
F^{+}(x)+F^{-}(x) & =0, & & x \in E,
\end{aligned}
$$

where $F^{+}$and $F^{-}$are defined by (29). In case $E=(-\infty, \infty)$ only the first equation in (40) is relevant, and when $g=0$ the equation is satisfied whenever $F^{-}=0$, i.e., whenever $f \in L_{p}^{+}$. Assume now that $E$ is a proper subset of $(-\infty$, $\infty)$, that is, assume the complement of $E$ has positive measure. If $g=0$ then $F^{-}=0$ on $E$ and so everywhere; therefore $F^{+}=0$ on the complement of $E$ and so everywhere. Therefore $f=0$. Thus $T-I$ ( $I=$ identity operator) is one-one. Now the adjoint of $T-I$ on $L_{p}$ is just $T-I$ on $L_{q}$, and since the latter operator is one-one the former has dense range. It remains to find a $g$ for which (40) is not solvable. We take any $g \in L_{p}(E)$ which vanishes on a subset $E_{1}$ of $E$ of positive measure, but which does not vanish identically on $E$. Then since $F^{-}$belongs to $L_{p}^{-}$and vanishes on $E_{1}$ it must vanish identically. Then also $g$ vanishes on $E$, which is a contradiction.

Our purpose in this section is to find out something of the nature of the spectrum of $T$ for an arbitrary $E$. Our results along this line are far from complete, but Theorem IV below shows that generally the spectrum can be neither very large nor very small. In particular we shall see that for $p=2$ and for $E$ not the entire real line the spectrum is always the interval $[-1,1]$.

For a given $p>1$ denote by $\mathfrak{A}_{p}$ the circular arc with end-points \pm 1 and which passes through $i \cot \pi / p$, and by $\Re_{p}$ the closed region bounded by $\mathfrak{A}_{p}$ and $\mathfrak{A}_{q}\left(=-\mathfrak{A}_{p}\right)$. Note that $\mathfrak{R}_{p}=\mathfrak{R}_{q}$.

TheOREM IV. (a) The spectrum of $T$ is contained in $\Re_{p}$.

(b) (i) If $E=(-\infty, \infty)$ the spectrum consists of the points \pm 1 .

(ii) If $E$ is a semi-infinite interval $(a, \infty)$ the spectrum is $\mathfrak{A}_{q}$, and if $E$ is a semi-infinite interval $(-\infty, a)$ the spectrum is $\mathfrak{A}_{p}$.

(iii) In all other cases the spectrum contains $\mathfrak{A}_{p} \cup \mathfrak{A}_{q}$. 
Proof. (a) The arc $\mathfrak{A}_{p}$ consists of those $\lambda$ for which

$$
\arg \frac{\lambda-1}{\lambda+1}=\frac{2 \pi}{p}
$$

and similarly for $\mathfrak{A}_{q} ; \lambda \notin \Re_{p}$ means that for some determination $\beta$ of $\arg (\lambda-1)(\lambda+1)^{-1}$,

$$
|\beta|<2 \pi \min \left(p^{-1}, q^{-1}\right)
$$

In the latter case set

$$
\begin{aligned}
& G^{+}(x)=\exp \left(\frac{\beta}{2 \pi} \int_{E} \frac{(1+y x) d y}{(y-x)\left(1+y^{2}\right)}+\frac{1}{2} i \beta \chi_{E}(x)\right), \\
& G^{-}(x)=\exp \left(-\frac{\beta}{2 \pi} \int_{E} \frac{(1+y x) d x}{(y-x)\left(1+y^{2}\right)}+\frac{1}{2} i \chi_{X}(x)\right) .
\end{aligned}
$$

Then it follows from Lemma 5 that $G^{+} \in R_{q}^{+}, 1 / G^{-} \in \mathbb{R}_{q}^{-}$, and $G^{-} \in W_{p}$. By Theorem $I$ the operator $G$, and so also $T-\lambda I$, is invertible.

This completes the proof of part (a). Before going on to (b) we shall need a lemma.

Lemma 10. Assume the function $f$ defined on $(-\infty, \infty)$ takes only the two values $\pm \pi / 2$. If we set

$$
g(x)=\frac{1}{\pi} \int_{-\infty}^{\infty} \frac{(1+t x) f(t)}{(t-x)\left(1+t^{2}\right)} d t
$$

and if we have

$$
\int_{-T}^{T} e^{\theta(t)} d t<\infty
$$

for all finite $T$, then $f=\pi\left\{\chi_{(-\infty, a)}-1 / 2\right\}$ for some $a$ in $[-\infty, \infty]$.

Proof. Consider the analytic function

$$
h(z)=i \exp \frac{1}{\pi} \int_{-\infty}^{\infty} \frac{(1+t z) f(t)}{(t-z)\left(1+t^{2}\right)} d t, \quad g z>0 .
$$

We have

$$
\begin{aligned}
\arg h(x+i y) & =\frac{\pi}{2}+\frac{1}{\pi} g \int_{-\infty}^{\infty}\left\{\frac{1}{t-(x+i y)}-\frac{t}{1+t^{2}}\right\} f(t) d t \\
& =\frac{\pi}{2}+\frac{1}{\pi} \int_{-\infty}^{\infty} \frac{y}{(t-x)^{2}+y^{2}} f(t) d t
\end{aligned}
$$


Since $|f| \leqq \pi / 2$ we conclude that $0 \leqq \arg h(x+i y) \leqq \pi$, so that $h(z)$ has nonnegative imaginary part in the upper half-plane. By a theorem of Nevanlinna the most general such function has the representation

$$
h(z)=\alpha z+\beta+\int_{-\infty}^{\infty} \frac{1+t z}{t-z} d \mu(t)
$$

where $\mu$ is a bounded and nondecreasing function on $(-\infty, \infty), \alpha \geqq 0$, and $\beta$ is real. Denoting by $h(x)$ the boundary function of $h(z)$,

$$
h(x)=\lim _{y \rightarrow 0+} h(x+i y),
$$

we obtain from (43) and (44) respectively

$$
\begin{aligned}
& h(x)=i \exp [g(x)+i f(x)], \\
& h(x)=\alpha x+\beta+\int_{-\infty}^{\infty} \frac{1+t x}{t-x} d \mu(t)+i \pi \mu^{\prime}(x),
\end{aligned}
$$

the integral in (46) being interpreted as a principal value.

Now for any $p<1$

$$
\int_{-\infty}^{\infty}\left|h(x+i y)^{p}-h(x)^{p}\right| \frac{d x}{1+x^{2}} \rightarrow 0
$$

as $y \rightarrow 0$, where the arguments of the $p$ th powers are taken to lie between zero and $p \pi$. This follows from the fact that by means of the substitution $z=i(1-\zeta)(1+\zeta)^{-1}$ the function $h(z)^{p}$ is transformed into a function in $H_{1}$ of the unit circle. (See the proof of Lemma 5 above.) Let $x_{0}$ and $x_{1}$ be such that the limits of $h\left(x_{0}+i y\right)$ and $h\left(x_{1}+i y\right)$ exist as $y \rightarrow 0$. Then we certainly have

$$
\int_{x_{0}}^{x_{1}}\left|h(x+i y)^{p}-h(x)^{p}\right| d x \rightarrow 0
$$

as $y \rightarrow 0$, and so using Cauchy's theorem we deduce

$$
\int_{x_{0}}^{x_{1}} h(x)^{p} d x=\int_{x_{0}}^{x_{0}+i y} h(z)^{p} d z+\int_{x_{0}}^{x_{1}} h(x+i y)^{p} d x+\int_{x_{1}+i y}^{x_{1}} h(z)^{p} d z
$$

for any $y>0$. In all four integrals we may take the limit as $p \rightarrow 1$ under the integral signs. For the first, this follows from the fact that $h(x)^{p} \leqq e^{o(x)}+1$. which belongs to $L_{1}\left(x_{0}, x_{1}\right)$ by hypothesis; the integrand in the third integral is continuous, and by our assumption on $x_{0}$ and $x_{1}$ the same is true of the remaining two integrals. We conclude that (47) holds with $p=1$. Next taking $y$ to zero we obtain

$$
\int_{x_{0}}^{x_{1}} h(x) d x=\lim _{\nu \rightarrow 0} \int_{x_{0}}^{x_{1}} h(x+i y) d x .
$$


Now since $f= \pm \pi / 2$ it follows from (45) that $h(x)$ is purely real. Taking imaginary parts in (48) and using (44) we deduce

$$
\begin{aligned}
0 & =\lim _{y \rightarrow 0} \int_{x_{0}}^{x_{1}}\left\{\alpha y+y \int_{-\infty}^{\infty} \frac{t^{2}+1}{(t-x)^{2}+y^{2}} d \mu(t)\right\} d x \\
& =\lim _{y \rightarrow 0} \int_{-\infty}^{\infty}\left(t^{2}+1\right) d \mu(t) \int_{x_{0}}^{x_{1}} \frac{y}{(t-x)^{2}+y^{2}} d x \\
& =\pi \int_{x_{0}}^{x_{1}}\left(t^{2}+1\right) d \mu(t)
\end{aligned}
$$

if $\mu$ is suitably normalized. Since $x_{0}$ can be taken arbitrarily close to $-\infty$ and $x_{1}$ to $+\infty$, we may conclude that $\mu$ is constant. Therefore $h(z)=\alpha z+\beta$, and the conclusion of the lemma with $a=-\beta / \alpha$ is now immediate from (45).

Going back to the proof of part (b) of Theorem IV, we see that (i) is immediate, for in this case $T^{2}=I$. Part (ii) will follow from Theorem III but we defer this computation. We pass therefore to (iii). For $\lambda \in \Re_{p}$ we have shown that $(T-\lambda I)^{-1}$ exists; let us explicitly solve $T f=\lambda f+g$ for such $\lambda$. Recall that $\beta$ denotes that $\arg (\lambda-1)(\lambda+1)^{-1}$ which satisfies (41). Then if $G^{+}$and $G^{-}$are given by (42) the solution of $T f=\lambda f+g$ is $f=f_{+}+f_{-}$where

$$
f_{+}=-\frac{1}{\lambda+1} \frac{1}{G^{+}}\left(\frac{g}{G^{-}}\right)_{+}, \quad f_{-}=-\frac{1}{\lambda+1} G^{-}\left(\frac{g}{G^{-}}\right) .
$$

Since $\left\|f_{+}\right\|_{p} \leqq A\|f\|_{p}$ we see that

$$
\left\|\frac{1}{G^{+}}\left(\frac{g}{G^{-}}\right)_{+}\right\|_{p} \leqq A\left\|(T-\lambda I)^{-1}\right\|\|g\|_{p}
$$

where $A$ is bounded for $\lambda$ not near -1 . Since $1 / G^{+} G^{-}=1 / G$ is bounded for $\lambda$ not near 1 (see (39)) we obtain

$$
\left\|G^{-}\left(\frac{g}{G^{-}}\right)_{+}\right\|_{p} \leqq A\left\|(T-\lambda I)^{-1}\right\|\|g\|_{p}
$$

as long as $\lambda$ is not near \pm 1 and $g$ vanishes outside $E$. It follows easily that

$$
\left\|G^{-} \tilde{g}\right\|_{p} \leqq A\left\|(T-\lambda I)^{-1}\right\|\left\|G^{-} g\right\|_{p}
$$

for all $g$ vanishing outside $E$ and for which $g(x) /(1+|x|) \in L_{1}$, as long as $\lambda$ is not near \pm 1 . Set

$$
\xi(x)=\exp \frac{1}{2} \int_{E} \frac{(1+t x) d t}{(t-x)\left(1+t^{2}\right)} .
$$

Then (49) says 


$$
\left\|\xi^{-\beta / \pi} \tilde{g}\right\|_{p} \leqq A\left\|(T-\lambda I)^{-1}\right\|\left\|\xi^{-\beta / \pi} g\right\|_{p} .
$$

Assume now that $\lambda_{0} \in \mathfrak{A}_{p} \cup \mathfrak{A}_{q}$ and that $\lambda_{0}$ does not belong to the spectrum of $T$, and let $\lambda \rightarrow \lambda_{0}$ from outside $\Re_{p}$. Assuming, as we may, that $p \geqq 2$ (considering the adjoint of $T-\lambda I$ shows that the theorem for $p \geqq 2$ yields the theorem for $p \leqq 2$ ), the $\beta$ corresponding to $\lambda$ approaches $\pm 2 \pi / p$ depending on whether $\lambda_{0}$ belongs to $\mathfrak{A}_{p}$ or $\mathfrak{A}_{q}$. Moreover $\left\|(T-\lambda I)^{-1}\right\|$ remains bounded so (50) gives, with the appropriate sign taken,

$$
\left\|\xi^{ \pm 2 / p} \tilde{g}\right\|_{p} \leqq A\left\|\xi^{ \pm 2 / p} g\right\|_{p}
$$

as long as $g$ vanishes outside $E$. Thus $\xi^{ \pm 2 / p} \in W_{p, E,(-\infty, \infty)}$, so by Lemma 2,

$$
\int_{-\infty}^{\infty} \frac{\xi(x)^{ \pm 2}}{1+|x|^{p}} d x<\infty
$$

By Lemma $10, E$ must be $(-\infty, \infty)$ or a semi-finite interval, and (iii) is proved.

REMARK 1. In the discussion immediately preceding, the " + " sign corresponded to $\lambda_{0} \in \mathfrak{P}_{q}$. If $\lambda_{0}$ does not belong to the spectrum of $T$ then Lemma 10 tells us, not only that $E$ is a semi-infinite interval, but that it is of the form $(-\infty, a)$. Thus if $E=(a, \infty)$ the spectrum contains $\mathfrak{A}_{q}$; and similarly if $E=(-\infty, a)$ the spectrum contains $\mathfrak{A}_{p}$. This is half of part $(b, \mathrm{ii})$ of Theorem IV.

Using Theorem III the spectrum of $T$ is easily computed in case $E$ is a finite union of (finite or infinite) intervals. We shall consider here only the two simplest cases, a semi-finite interval (which will finally complete part (b, ii) of Theorem IV) and a finite interval.

(1) $E=(0, \infty)$. Assume first that $p \geqq 2$ and that $\lambda$ belongs to the interior of $\Re_{p}$ or of $\mathfrak{R}_{p .}$. Then we can choose an argument $\beta$ of $(\lambda-1)(\lambda+1)^{-1}$ which satisfies $2 \pi p^{-1} \leqq \beta<2 \pi q^{-1}$. In the notation of Theorem II we have $a_{0}=-\beta$, $a_{1}=\beta ; c_{0}=-\beta, c_{1}=\beta ; b_{0}=b_{1}=0$. Thus $m=0$ and by Theorem III $\lambda$ is not in the spectrum of $T$. By Remark 1 above the spectrum contains $\mathfrak{A}_{q}$ and so this is exactly the spectrum. Using the fact that $T$ is "self-adjoint" the same result holds for $p \leqq 2$.

(2) $E=(\mu, \nu)$ with $-\infty<\mu<\nu<\infty$. Again let $p \geqq 2$ and assume $\lambda$ belongs to the interior of $\Re_{p}$. Choose the argument $\beta$ of $(\lambda-1)(\lambda+1)^{-1}$ which satisfies $2 \pi p^{-1}<\beta<2 \pi q^{-1}$. Then $a_{0}=0, a_{1}=\beta, a_{2}=-\beta ; c_{0}=0, c_{1}=\beta, c_{2}=2 \pi-\beta ; b_{0}=b_{1}$ $=0, b_{2}=-2 \pi$. Thus $\lambda$ belongs to the residual spectrum of $T$ and the range of $T-\lambda I$ has deficiency one. The spectrum of $T$ is $\Re_{p}$. In case $p \leqq 2$ the spectrum is again $\Re_{p}$; but every $\lambda$ in the interior of $\Re_{p}$ is an eigenvalue of multiplicity one, and $T-\lambda I$ is onto.

REMARK 2. By the above method one can determine, in case $E$ is a finite union of intervals, whether a given $\lambda$ in the interior of $\Re_{p}$ belongs to the resolvent set, point spectrum, or residual spectrum of $T$. (It will always 
be one of these.) One may well wonder what happens if $\lambda \in \mathfrak{A}_{p} \cup \mathfrak{A}_{q}$. We have already shown that \pm 1 belong to the continuous spectrum. More generally if $\lambda \in \mathfrak{A}_{p} \cup \mathfrak{A}_{q}$ and $\lambda$ is in the spectrum, it will be in the continuous spectrum. To give the idea of the proof we shall consider the case $\lambda=0, p=2, E=(0, \infty)$. By self-adjointness it suffices to show $T f=0$ implies $f=0$. We obtain $G f_{+}$ $=-f_{-}$, where $G(x)=-1$ for $x>0$ and $G(x)=1$ for $x<0$. Write $G=G^{+} G^{-}$ where

$$
\begin{array}{ll}
G^{+}(x)=x^{1 / 2}, & (\arg x=0, \pi), \\
G^{-}(x)=x^{-1 / 2}, & (\arg x=\pi, 2 \pi) .
\end{array}
$$

Then $G^{+} f_{+}=-f_{-} / G^{-}$. Using Carleman's theorem and (4) we deduce that both sides are of the form $c x$, so $f(x)=2 c x^{1 / 2}$, which does not belong to $L_{2}$ unless $c=0$.

REMARK 3. In case $E$ is a finite union of intervals, but is neither $(-\infty, \infty)$ nor a semi-finite interval, it turns out that the spectrum of $T$ is all of $\Re_{p}$. This supports the conjecture that part (b, iii) of Theorem IV may be strengthened to read: In all other cases the spectrum is $\Re_{p}$.

Added in proof. We have recently proved the conjecture for $E$ bounded.

6. Reduction of singular integral equations in $L_{p}$. We come now to the equation

$$
K f(x) \equiv A(x) f(x)+\frac{B(x)}{\pi i} \int_{E} \frac{f(x)}{y-x} d y+M f(x)=g(x)
$$

where $M$ is a completely continuous operator on $L_{p}(E)$, and $A$ and $B$ satisfy the usual conditions: $A$ and $B$ are bounded and $A^{2}-B^{2}$ is bounded away from zero. Write

$$
A_{1}(x, y)=\frac{A(y) A(x)^{-1}-1}{y-x}, \quad B_{1}(x, y)=\frac{\tilde{B}(y)-\tilde{B}(x)}{y-x},
$$

where

$$
\tilde{B}(x)=\frac{1}{\pi} \int_{E} \frac{B(y)}{y-x} d y .
$$

The function $G(x)$ is defined by (33).

Theorem V. Suppose $G(x)$ satisfies the conditions $1^{\circ}$ and $2^{\circ}$ of $\S 3$, and let $a_{k}, b_{k}$, and $c_{k}$ be as defined there. Assume each $\left|c_{k}\right|<2 \pi \min \left(p^{-1}, q^{-1}\right) ;$ assume further that

$$
\int_{E}\left|A_{1}(x, y)\right|^{q} d y \in L_{p / q}(E), \quad \int_{E}\left|B_{1}(x, y)\right|^{q} d y \in L_{p / q}(E) .
$$


Then the equation (51) is equivalent, in the sense described below, to an equation of the form $(I+N) \phi=\psi$ where $N$ is completely continuous. by

Proof. Let $\boldsymbol{K}_{0}$ be the operator defined by (2), and $\boldsymbol{K}_{0}^{\prime}$ the operator defined

$$
\boldsymbol{K}_{0}^{\prime} f(x) \equiv A(x) f(x)-\frac{B(x)}{\pi i} \int_{E} \frac{f(y)}{y-x} d y
$$

We have

$$
\begin{aligned}
K_{0}^{\prime} K_{0} f(x)= & A(x)^{2} f(x)+\frac{A(x) B(x)}{\pi i} \int_{E} \frac{f(y)}{y-x} d y \\
& -\frac{B(x)}{\pi i}\left\{\int_{E} \frac{A(y) f(y)}{y-x} d y+\frac{1}{\pi i} \int_{E} \frac{B(t)}{t-x} d t \int_{E} \frac{f(y)}{y-t} d y\right\} .
\end{aligned}
$$

The Poincaré-Bertrand formula

$$
\int \frac{B(t)}{t-x} d t \int \frac{f(y)}{y-t} d y=-\pi^{2} B(x) f(x)+\int f(y) d y \int \frac{B(t)}{(t-x)(y-t)} d t
$$

(which holds whenever $f \in L_{p}$ and $B \in L_{q}[5]$ ), together with (52), gives

$$
\begin{aligned}
K_{0}^{\prime} K_{0} f(x)= & \left\{A(x)^{2}-B(x)^{2}\right\} f(x)-\frac{A(x) B(x)}{\pi i} \int_{E} A_{1}(x, y) f(y) d y \\
& -\frac{1}{\pi} \int_{E} B_{1}(x, y) f(y) d y .
\end{aligned}
$$

Define the operator $P$ by

$$
P f(x) \equiv \frac{A(x) B(x)}{\pi i} \int_{E} A_{1}(x, y) f(y) d y+\frac{1}{\pi} \int_{E} B_{1}(x, y) f(y) d y .
$$

Then (53) implies that $\boldsymbol{P}$ is completely continuous. Moreover

$$
\boldsymbol{K}_{0}^{\prime} \boldsymbol{K}_{0} f(x)=\left\{A(x)^{2}-B(x)^{2}\right\} f(x)-P f(x) .
$$

Since $K_{0}$ and $K_{0}^{\prime}$ are interchanged when we change the sign of $B$, we also obtain

$$
\boldsymbol{K}_{0} K_{0}^{\prime} f(x)=\left\{A(x)^{2}-B(x)^{2}\right\} f(x)+P f(x) .
$$

It follows from our assumption on the $c_{k}$ that if

$$
m=\frac{1}{2 \pi} \sum_{k=0}^{n} b_{k}
$$


then the " $m$ " for $K_{0}^{\prime}$ is just $-m$. We therefore consider two cases.

(1) $m \geqq 0$. Then $K_{0}^{\prime}$ is one-one. Multiplying both sides of (51) on the left by $\boldsymbol{K}_{0}^{\prime}$ gives

$$
K_{0}^{\prime} K_{0} f+K_{0}^{\prime} M f=K_{0}^{\prime} g
$$

Setting

$$
N f(x) \equiv-\frac{P f(x)}{A(x)^{2}-B(x)^{2}}+\frac{K_{0}^{\prime} M f(x)}{A(x)^{2}-B(x)^{2}}
$$

and

$$
\psi(x)=\frac{K_{0}^{\prime} g(x)}{A(x)^{2}-B(x)^{2}}
$$

we see from (54) that (56) is equivalent to

$$
(I+N) f=\psi
$$

which is an equation of the required type. Moreover if (57) holds then so does (56), since they are identical except for notation, and this in turn implies $\boldsymbol{K}_{0} f+\boldsymbol{M} f=\boldsymbol{g}$ because $\boldsymbol{K}_{0}^{\prime}$ is one-one.

(2) $m \leqq 0$. Then $K_{0}^{\prime}$ is onto. Let us set

$$
f=K_{0}^{\prime} \phi \text {. }
$$

Then (51) becomes

$$
K_{0} K_{0}^{\prime} \phi+M K_{0}^{\prime} \phi=g .
$$

Setting

$$
N \phi(x) \equiv \frac{P \phi(x)}{A(x)^{2}-B(x)^{2}}+\frac{M K_{0}^{\prime} \phi(x)}{A(x)^{2}-B(x)^{2}}
$$

and

$$
\psi(x)=\frac{g(x)}{A(x)^{2}-B(x)^{2}}
$$

we see from (55) that (59) is equivalent to

$$
(I+N) \phi=\psi \text {. }
$$

Moreover if (60) holds then so does (59), and defining $f$ by (58) we obtain (51).

\section{REFERENCES}

1. T. Carleman, L'integrale de Fourier et questions qui s'y rattachent, Uppsala, Almquist \& Wiksells, 1944.

2. G. H. Hardy, J. E. Littlewood, and G. P6lya, Inequalities, Cambridge, Cambridge University Press, 1934. 
3. E. Hille and J. D. Tamarkin, On the absolute integrability of Fourier transforms, Fund. Math. vol. 25 (1935) pp. 329-352.

4. I. I. Hirschman, Jr., Decomposition of Walsh and Fourier series, Memoirs Amer. Math. Soc. no. 15, 1955.

5. B. V. Hvedelidze, Some properties of improper integrals in the sense of the Cauchy-Lebesgue principal value, Soobšx. Akad. Nauk Gruzin. SSR. vol. 8 (1947) pp. 283-290.

6. H. Kober, A note on Hilbert's operator, Proc. Amer. Math. Soc. vol. 48 (1942) pp. 421427.

7. L. H. Loomis, An introduction to abstract harmonic analysis, New York, Van Nostrand, 1953.

8. N. I. Muskhelishvili, Singular integral equations, Groningen, P. Noordhoff, 1953.

9. E. M. Stein, Interpolation of linear operators, Trans. Amer. Math. Soc. vol. 83 (1956) pp. $482-492$.

10. G. Szegö, Über die Randwerte analytischer Functionen, Math. Ann. vol. 84 (1921) pp. 232-244.

11. E. C. Titchmarsh, Introduction to the theory of Fourier integrals, Oxford, Oxford University Press, 1937.

12. A. Zygmund, Sur les fonctions conjugies, Fund. Math. vol. 13 (1929) pp. 284-303.

13. — Trigonometrical series, New York, Chelsea Publishing Co., 1952.

\section{Cornell UNIVERsity,}

ITHACA, NEW YORK 